\title{
IMPACT OF DIABETES ON OUTCOMES OF CARDIAC SURGERY IN SOUTHEAST ASIAN PATIENTS
}

\author{
Vikaesh Moorthy', Weiling Liu'2, Lian Kah Ti1,2, Sophia Chew Tsong Huey ${ }^{3,4}$ \\ ${ }^{1}$ National University of Singapore, Yong Loo Lin School of Medicine, ${ }^{2}$ National University Hospital, Department of Anaesthesia, ${ }^{3}$ Singapore General Hospital, \\ Department of Anaesthesiology, ${ }^{4}$ Duke-NUS Graduate Medical School, Department of Cardiovascular and Metabolic Disorders
}

\section{INTRODUCTION}

Diabetes mellitus is a major health problem worldwide today with Asia having the greatest disease burden. Although diabetes is rapidly increasing in Asia and has been shown to be associated with worse cardiac surgery outcomes, no research has been done to study the impact of diabetes on cardiac surgery outcomes in a Southeast Asian cohort [1]. Hence, this study aims to delineate the impact of diabetes in a multi-ethnic Southeast Asian cohort undergoing cardiac surgery.

\section{METHODS}

We analysed data from 2831 adult patients undergoing elective cardiac surgery, from 2008 to 2010 at the two main heart centres in Singapore. Perioperative variables and outcomes of diabetics and nondiabetics were compared.

\section{RESULTS}

Patients with diabetes tended to be older, more obese, Indian, hypertensive and have renal impairment. Compared to nondiabetics, diabetics had a higher relative higher risk for postoperative hyperglycemia (RR 1.575, $\mathrm{Cl} 1.500-1.654)$, AKI (RR 1.419, Cl 1.269 - 1.587), new need for dialysis (RR 2.397, 1.573 - 3.653), postoperative infection (RR 2.110, Cl 1.231 - 3.617) and ICU readmission (RR 1.768, Cl 1.253 - 2.495) (Table 1).

\begin{tabular}{|c|c|c|c|c|c|}
\hline Outcome & $\begin{array}{l}\text { No. of } \\
\text { events }\end{array}$ & $\begin{array}{c}\text { Absolute risk/ } 1000 \\
\text { patients }\end{array}$ & Relative Risk (95\% Cl) & $\begin{array}{l}\text { Relative Risk }(95 \% \mathrm{Cl} \text { ) in } \\
\text { Western Cohort Study }\end{array}$ & Ref \\
\hline \multicolumn{6}{|l|}{ Postoperative } \\
\hline Hyperglycemia & 846 & 299 & 1.000 & - & - \\
\hline Nondiabetic & 1166 & 412 & $1.575(1.500-1.654)$ & & \\
\hline \multicolumn{6}{|l|}{ Diabetic } \\
\hline \multicolumn{6}{|l|}{ AKI } \\
\hline Nondiabetic & 386 & 136 & 1.000 & 1.000 & [1] \\
\hline Diabetic & 479 & 169 & $1.419(1.269-1.587)$ & $1.43(1.26-1.61)$ & \\
\hline \multicolumn{6}{|l|}{ New dialysis } \\
\hline Nondiabetic & 31 & 11 & 1.000 & - & - \\
\hline Diabetic & 65 & 23 & $2.397(1.573-3.653)$ & & \\
\hline \multicolumn{6}{|c|}{ Postoperative Infection } \\
\hline Nondiabetic & 20 & 7 & 1.000 & 1.000 & [2] \\
\hline Diabetic & 37 & 13 & $2.110(1.231-3.617)$ & $2.03(1.13-4.05)$ & \\
\hline \multicolumn{6}{|l|}{ ICU readmission } \\
\hline Nondiabetic & 51 & 18 & 1.000 & 1.000 & [3] \\
\hline Diabetic & 79 & 28 & $1.768(1.253-2.495)$ & $1.39(1.17-1.66)$ & \\
\hline
\end{tabular}

Table 1 Relative risks for clinical outcomes in Southeast Asian diabetics compared to similar Western cohorts. AKI, Acute Kidney Injury; ICU, Intensive Care Unit.

\section{CONCLUSION}

In conclusion, diabetes is associated with increased risk for renal dysfunction, hyperglycemia and infection after cardiac surgery as compared to nondiabetics, similar to the relative risks of diabetics observed in Western populations. As such, diabetic patients undergoing cardiac surgical interventions should receive special attention during the perioperative period to prevent the identified adverse effects, thereby improving postoperative outcomes of diabetic patients.

\section{REFERENCES}

1. Bucerius J et al. Thorac Cardiovasc Surg. 2003;51(1):11-16

2. Ponzoni CR et al. Ann Am Thorac Soc. 2017;14(8):1312-1319

3. Haffner SM. Diabetes Care. 1998;21 Suppl 3:C3-6. 